\title{
Proton-Coupled Electron Transfer in the Reaction of 3,4-Dihydroxyphenylpyruvic Acid with Reactive Species in Various Media
}

\author{
J. J. Fifen, ${ }^{1}$ Z. Dhaouadi, ${ }^{2}$ M. Nsangou, ${ }^{3}$ O. Holtomo, ${ }^{4}$ and N. Jaidane ${ }^{2}$ \\ ${ }^{1}$ Department of Physics, Faculty of Science, The University of Ngaoundere, P.O. Box 454, Ngaoundere, Cameroon \\ ${ }^{2}$ Laboratoire de Spectroscopie Atomique, Moléculaire et Applications, Faculté des Sciences de Tunis, Université de Tunis El Manar, \\ Campus Universitaire, 1060 Tunis, Tunisia \\ ${ }^{3}$ University of Maroua, P.O. Box, 46 Maroua, Cameroon \\ ${ }^{4}$ Fundamental Physics Lab, Graduate Training Unit in Physics and Engineering Sciences, Faculty of Science, University of Douala, \\ P.O. Box 24157, Douala, Cameroon
}

Correspondence should be addressed to J. J. Fifen; julesfifen@gmail.com

Received 26 August 2014; Revised 21 December 2014; Accepted 22 December 2014

Academic Editor: Franck Rabilloud

Copyright (C) 2015 J. J. Fifen et al. This is an open access article distributed under the Creative Commons Attribution License, which permits unrestricted use, distribution, and reproduction in any medium, provided the original work is properly cited.

\begin{abstract}
The distinction of concerted proton-coupled electron transfer (CPCET) from sequential one as well as proton transfer-electron transfer (PT-ET) from electron transfer-proton transfer (ET-PT) in the $\mathrm{O}-\mathrm{H}$ bond cleavage reactions in various media has always been a difficult task. In this work, the activation barrier of the CPCET mechanism, its rate constants, and reaction free energies related to ET-PT and PT-ET involving coreactive species were presented as good parameters to attempt the problem. DFT calculations were carried out studying the described pathways subsequent to the scavenging of ${ }^{\circ} \mathrm{OH}$ and $\mathrm{OBr}^{-}$by the $3,4-$ DHPPA in various media. The solvation was described in a hybrid manner using IEF-PCM model conjointly with a model that takes into account some solute-solvent interactions. As a result, we found that the scavenging of hydroxyl radical by 3,4-DHPPA is thermodynamically governed by a one-step hydrogen atom transfer (CPCET) from the acid to the radical in all media. In kinetic viewpoint, CPCET still dominates in the vacuum and in nonpolar solvents, but in polar solvents it could compete strongly with the ET-PT mechanism so that the latter could slightly dominate.
\end{abstract}

\section{Introduction}

Understanding proton transfer (PT) or proton-coupled electron transfer (PCET) mechanism is very important in chemical processes. Such mechanisms can be subsequent to the $\mathrm{O}-$ $\mathrm{H}$ bond cleavage. The $\mathrm{O}-\mathrm{H}$ bond cleavage subsequent to a transfer of the proton or a hydrogen atom from the concerned molecule to another molecule is governed by two main mechanisms: proton-coupled electron transfer (PCET) and proton transfer process (PT). In contrast to simple electron transfer (ET) or proton transfer (PT) reactions, PCET is more complex as both an electron and a proton must be transferred and their coupling strongly influences the process thermodynamically and kinetically. In PCET, the transfer of an electron and a proton may be sequential (stepwise) or concerted. In sequential transfer, either the electron or proton is transferred first. When the electron is transferred first, the process is termed electron transfer-proton transfer (ETPT); otherwise, it is termed proton transfer-electron transfer (PT-ET) $[1,2]$. In the concerted mechanism, the electron and proton are transferred simultaneously, and this is termed concerted proton-coupled electron transfer (CPCET) $[1,2]$.

Usually, theoretically or experimentally, it is very difficult to distinguish CPCET from PT-ET or ET-PT. It is quite always an open problem. Recently, we studied the 3,4-dihydroxyphenylpyruvic acid (3,4-DHPPA) O-H bond cleavage in various media [3]. The scheme used in such a work does not consider the coreactive species (molecules or atoms which receive the transferred proton or hydrogen atom). It is only based on the calculated bond dissociation enthalpy 
(BDE), proton affinity $(\mathrm{PA})$, and proton dissociation enthalpy (PDE). The main result derived in that work is that solvent notably influences and changes the nature of hydrogen atom cleavage, favoring by this way the proton transferelectron transfer (PT-ET) mechanism in polar solvents and the concerted proton-coupled electron transfer (CPCET) mechanism in nonpolar solvents. Using the same scheme, the same results were pointed out by several other authors [4-9], studying the hydrogen atom cleavage on different systems in various media. The question is, do we have a complete insight into such mechanisms without taking into account coreactive species?

Aiming to provide a reliable answer to the above question, a theoretical scheme involving reaction free energies (RFEs) related to the described processes was presented and the study is completed by a kinetic investigation. The application case concerns the $\mathrm{O}-\mathrm{H}$ bond cleavage subsequent to the scavenging of ${ }^{\circ} \mathrm{OH}$ and $\mathrm{OBr}^{-}$by the 3,4-dihydroxyphenylpyruvic acid (3,4-DHPPA) in the vacuum, cyclohexane, benzene, DMSO, and water. Solvents were chosen in regard to their increasing polarity or dielectric constant.

3,4-DHPPA is a phenolic acid (PhA) reported as an alternative substrate for mammalian 4-hydroxyphenylpyruvate dioxygenase, which gives competitive inhibition versus 4hydroxyphenylpyruvate $[10,11]$. PhAs and their derivatives are widely present in plants (vegetables, fruits, grains, and spices), many being metabolites and several functions being attributed to them [12]. PhAs may contribute to the dark colour, bitter taste, and objectionable flavour of some fruits, leaves, and seeds. They have been considered as possible agents that influence toxicological, nutritional, sensory, and antioxidant properties of foods [12]. It has also been reported that phenolic acids may activate or inhibit microbial growth [12-16]. PhAs present antioxidant activity and exert this property through their capacity to donate hydrogen atoms, protons, and/or electrons to inhibit reactive oxygen species. The enol tautomer of 3,4-DHPPA, which was less studied as compared to the keto tautomer, was pointed out by Milane as being the fragment of Quercetin responsible for its higher antioxidant activity [17]. Thus, the results obtained involving 3,4-DHPPA could be generalized to Quercetin. Moreover, metal ions effects on the antioxidant activity of 3,4-DHPPA were studied in the vacuum [18]. A conformational study of this acid was also undertaken [3] and the likeliest conformer is depicted in Figure 1. The ${ }^{\circ} \mathrm{OH}$ radical has been chosen as the most unstable and the most reactive among the available reactive oxygen species (ROS), whereas $\mathrm{OBr}^{-}$is chosen as a nonradical oxygen species involved in several biological processes [19-21]. The thermodynamic study of the reaction of 3,4-DHPPA with the ${ }^{\circ} \mathrm{OH}$ radical was completed by a kinetic study involving tunnelling and recrossing effects.

\section{Computational Methods}

2.1. Calculations of Thermodynamic Parameters. When coreactive species are not taken into account, thermodynamic parameters characterizing PCET mechanism can be evaluated through bond dissociation free energy (BDFE), ioniza-

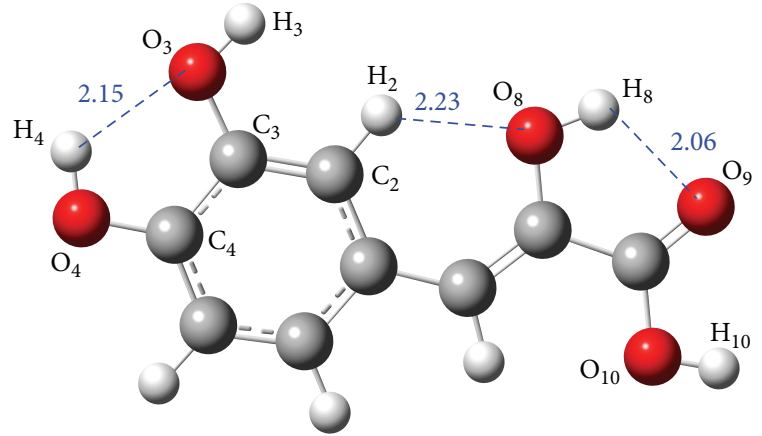

FIGURE 1: Equilibrium geometry of the likeliest conformer of 3,4DHPPA in the vacuum, $6-31+G(d)$.

tion potential (IP), proton dissociation free energy (PDFE), proton affinity (PA), and electron free energy (ETFE) described as follows:

(i) $\mathrm{BDFE}$ of a given $\mathrm{O}-\mathrm{H}$ bond is the free energy change of the dissociation reaction:

$$
\mathrm{RO}-\mathrm{H} \longrightarrow \mathrm{RO}^{\bullet}+{ }^{\bullet} \mathrm{H}
$$

$\mathrm{BDFE}=\Delta_{f} G^{\circ}\left(\mathrm{RO}^{\circ}\right)+\Delta_{f} G^{\circ}\left({ }^{\circ} \mathrm{H}\right)-\Delta_{f} G^{\circ}(\mathrm{RO}-\mathrm{H})$.

(ii) IP of the antioxidant is the free energy change required to remove its electron:

$$
\begin{gathered}
\mathrm{RO}-\mathrm{H} \longrightarrow(\mathrm{RO}-\mathrm{H})^{+\bullet}+e^{-} \\
\mathrm{IP}=\Delta_{f} G^{\circ}(\mathrm{RO}-\mathrm{H})^{+\bullet}+\Delta_{f} G^{\circ}\left(e^{-}\right)-\Delta_{f} G^{\circ}(\mathrm{RO}-\mathrm{H}) .
\end{gathered}
$$

(iii) PDFE is defined as the free energy change of the dissociation reaction:

$$
\mathrm{RO}-\mathrm{H}^{+\bullet} \longrightarrow \mathrm{RO}^{\bullet}+\mathrm{H}^{+}
$$

$\operatorname{PDFE}=\Delta_{f} G^{\circ}\left(\mathrm{RO}^{\bullet}\right)+\Delta_{f} G^{\circ}\left(\mathrm{H}^{+}\right)-\Delta_{f} G^{\circ}\left(\mathrm{RO}-\mathrm{H}^{+\bullet}\right)$.

(iv) $\mathrm{PA}$ of the anion $\mathrm{RO}^{-}$is the free energy change of the dissociation reaction:

$$
\begin{gathered}
\mathrm{RO}-\mathrm{H} \longrightarrow \mathrm{RO}^{-}+\mathrm{H}^{+} \\
\mathrm{PA}=\Delta_{f} G^{\circ}\left(\mathrm{RO}^{-}\right)+\Delta_{f} G^{\circ}\left(\mathrm{H}^{+}\right)-\Delta_{f} G^{\circ}(\mathrm{RO}-\mathrm{H}) .
\end{gathered}
$$

(v) ETFE represents the free energy change of the reaction:

$$
\mathrm{RO}^{-} \longrightarrow \mathrm{RO}^{\bullet}+e^{-}
$$

$$
\text { ETFE }=\Delta_{f} G^{\circ}\left(\mathrm{RO}^{\circ}\right)+\Delta_{f} G^{\circ}\left(e^{-}\right)-\Delta_{f} G^{\circ}\left(\mathrm{RO}^{-}\right) .
$$

When coreactive species are taken into account in the cleavage process, the mechanism can be presented as summarized in Figure 2 and the process is characterized by the reaction free energy (RFE). In such a scheme, RFE is 


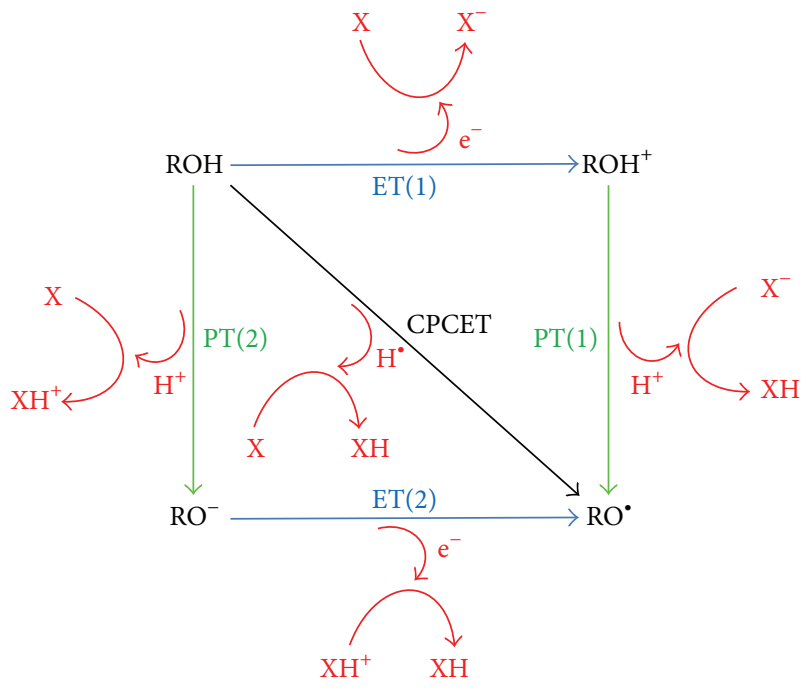

FIGURE 2: Thermodynamic cycle showing ET-PT, PT-ET, and CPCET mechanisms involving coreactive species X. The indices 1 and 2 are devoted to ET-PT and PT-ET mechanisms, respectively. $\mathrm{ROH}$ is a phenolic acid.

defined as the sum of free energies of the donor and acceptor reactions. For example, RFE related to the mechanism ET(1) in the scheme is denoted by RFE-ET(1) and calculated as

$$
\begin{aligned}
\operatorname{RFE}-E T(1)= & \Delta_{f} G_{\text {solv }}^{\circ}\left(\mathrm{ROH}^{+}\right)+\Delta_{f} G_{\text {solv }}^{\circ}\left(\mathrm{X}^{-}\right) \\
& -\Delta_{f} G_{\text {solv }}^{\circ}(\mathrm{ROH})-\Delta_{f} G_{\text {solv }}^{\circ}(\mathrm{X}) .
\end{aligned}
$$

According to the above thermodynamic cycle, the possibility of ET-PT and PT-ET mechanisms can be evaluated, respectively, through RFE-ET(1) and RFE-PT(2), since ET(1) and $\mathrm{PT}(2)$ are, respectively, the first paths of these mechanisms.

2.2. Rate Constants Computations. Rate constants are computed as a function of temperature $T$, in the zero-order semiclassical transition state theory [22]. Thus, the rate constant for the canonical transition state theory (TST) is given by

$$
k(T)=\kappa(T) \times k_{\mathrm{TST}}(T),
$$

where, for a bimolecular reaction as considered herein,

$$
k_{\mathrm{TST}}(T)=\frac{\sigma}{\beta h} \times \frac{Q^{\neq}(T)}{Q_{A}(T) Q_{B}(T)} \times e^{-\beta \Delta V^{\neq}} ;
$$

$k_{\mathrm{TST}}$ is the conventional TST rate constant at $T$ temperature, without tunnelling correction. $\beta=1 / k_{B} T ; Q_{A}(T), Q_{B}(T)$, and $Q^{\ddagger}(T)$ are, respectively, the partition functions of the reactants $A, B$ and the transition state at temperature T. $h$ and $k_{B}$ are, respectively, the Plank and Boltzmann constants. $\Delta V^{\neq}$ is the classical barrier height while $\sigma$ is the symmetry factor accounting for the possibility of two or more symmetryrelated reaction paths. $\kappa(T)$ is a ground state transmission coefficient at temperature $T$, which primarily accounts for tunnelling correction.

Usually, tunnelling is considered only in the degree of freedom corresponding to the reaction coordinate. Within this one-dimensional formalism, we have used two different approximations of the transmission coefficient. The first approximation is a truncated parabola for a potential energy through which tunnelling happens. Therefrom, the transmission coefficient is [23]

$$
\begin{aligned}
& \kappa(T) \\
& =\beta \sum_{n=0}^{\infty}(-1)^{n}\left\{\frac{1-\exp \left\{[\beta-(n+1) \alpha] \Delta V^{\ddagger}\right\}}{(n+1) \alpha-\beta}+\frac{1}{n \alpha+\beta}\right\},
\end{aligned}
$$

where $\beta=1 / k_{B} T, \alpha=1 / \hbar\left|\nu^{\ddagger}\right|$, and $\nu^{\ddagger}$ is the imaginary frequency associated with the TS structure. For special cases,

$$
\kappa(T)= \begin{cases}1+\frac{1}{24}\left|\frac{h v^{\ddagger}}{k T}\right|^{2}, & \alpha \gg \beta \\ \frac{\pi \beta \alpha}{\sin (\pi \beta \alpha)}+\frac{\beta}{\beta-\alpha} \times e^{(\beta-\alpha) \Delta V^{\ddagger}}, & \alpha \geq \beta \\ \frac{\beta}{\beta-\alpha} \times\left[e^{(\beta-\alpha) \Delta V^{\ddagger}}-1\right], & \alpha \leq \beta .\end{cases}
$$

The case $\alpha \gg \beta$ is known as the Wigner tunnelling approximation [24] and the associated transmission coefficient can be noted by $\kappa_{W}$. The two other cases $(\alpha \geq \beta$ and $\alpha \leq \beta)$ are, respectively, known as the first-case and the secondcase of Skodje and Truhlar tunnelling approximation [23], and the associated transmission coefficients can be noted, respectively, by $\kappa_{\mathrm{ST}}^{>}$and $\kappa_{\mathrm{ST}}^{<}$.

The second approximation of the transmission coefficient is based on a variational transition state theory (VTST). In this approximation, the transmission coefficient is a zeroorder interpolated approximation $[25,26]$. By zero-order, the authors mean that no $a b$ initio or DFT calculations at points other than reactants, saddle point, and products are available. In addition, tunnelling is assumed to occur along the minimum energy path (MEP) which is interpolated by an Eckart function [27]. Since one is not sure that the highest point in the MEP corresponds to the highest free energy, the variational TST rate constant is defined as

$$
k_{V T S T}(T)=\min _{s}\left[\frac{\sigma}{\beta h} \times \frac{Q^{\ddagger}(T, s)}{Q_{A}(T) Q_{B}(T)} \times e^{-\beta \Delta V^{\ddagger}(s)}\right],
$$

where $s$ is the distance from the generalized transition state along the MEP. This last approximation of the transmission coefficient is implemented in POLYRATE 2010-A [28] and termed zero-order interpolated variational transition state theory (IVTST-0) which is rigorously equivalent to the traditional transition state with zero-curvature tunnelling (TST/ZCT). The computations of all rate constants have been performed using our homemade Fortran program and POLYRATE 2010-A. 
2.3. DFT, Basis Sets, and Solvation Methods Description. Due to the solute cavity description, implicit solvation models cannot provide a good description for short range solutesolvent interactions. In this work, such interactions were considered explicitly and the solvation was treated in a hybrid manner considering some explicit interactions between the particle to be transferred (during the scavenging mechanism) and a solvent molecule. The rest of solvent was treated implicitly using integral equation formalism polarized continuum model (IEF-PCM) [29]. Thus, aiming to compute the reaction free energies in solvent, we strongly needed solvation free energies of ${ }^{\bullet} \mathrm{H}, \mathrm{H}^{+}$, and $e^{-}$. All free energy changes were computed at a temperature of $298.15 \mathrm{~K}$ and a pressure of $1 \mathrm{~atm}$. Geometry optimizations were carried out using the density functional theory (DFT) methods implemented in the GAUSSIAN03 computational package [30].

DFT was chosen because of the excellent compromise between the computational time and the description of the electronic correlation. Throughout our calculations, the B3LYP hybrid functional which consists of Becke's three parameters exact exchange functional (B3) [31] combined with the nonlocal gradient corrected correlation functional of Lee-Yang-Parr (LYP) [32] was used. For systems with old number of electrons (radicals involved in this work), we used the restricted open shells approach (ROB3LYP). The basis sets used in this work were $6-31+\mathrm{G}^{*}$ and $6-311++\mathrm{G}^{* *}$ [33-35]. The last one is used especially for solvation free energies of the proton and electron. Full geometry optimization was carried out without symmetry constraints up to convergence (largest component of nuclear gradient equal to $10^{-6}$ a.u./bohr and the change in total energy less than $10^{-7}$ a.u.). A subsequent vibrational frequency calculation was undertaken in order to confirm that the resulting optimized geometries correspond to minima (no negative frequencies) or first-order saddle points (one negative frequency) on the PES, as we were looking for equilibria or transition states, respectively. The natural bond orbital (NBO) technique [36, 37] was used for natural population analysis (NPA). All these calculations were undertaken in the vacuum and in polar and nonpolar solvents. Farther in the work, G3B3 method was used for counterpoise computations. G3B3 is the Gaussian-3 (G3) method using B3LYP (B3) structures and frequencies for very accurate energies computations $[38,39]$.

\section{Results and Discussion}

Throughout this work, the $\mathrm{O}-\mathrm{H}$ bond cleavage through the reactions of 3,4-DHPPA with ${ }^{\circ} \mathrm{OH}$ or $\mathrm{OBr}^{-}$was considered. In such reactions, the reactive species (RS) considered react with the acid through the phenolic hydrogen atoms (or protons) of the acid, that is, $\mathrm{H}_{3}\left(\right.$ or $\left.\mathrm{H}_{3}^{+}\right), \mathrm{H}_{4}\left(\right.$ or $\left.\mathrm{H}_{4}^{+}\right)$, and $\mathrm{H}_{8}$ (or $\mathrm{H}_{8}^{+}$), since phenolic hydrogen atoms (or protons) are more acidic than carboxylic ones [12, 40-42]. Thus, in regard to the diagram of Figure 2, $\mathrm{X}$ may be replaced by ${ }^{\circ} \mathrm{OH}$ or $\mathrm{OBr}^{-}$when the considered coreactive is ${ }^{\circ} \mathrm{OH}$ or $\mathrm{OBr}^{-}$, respectively. Figures 3 and 4 depict the optimized geometries of supermolecules resulting from the reactions of 3,4-DHPPA with ${ }^{\circ} \mathrm{OH}$ and $\mathrm{OBr}^{-}$at the $3-\mathrm{OH}$ attacked site in the vacuum,

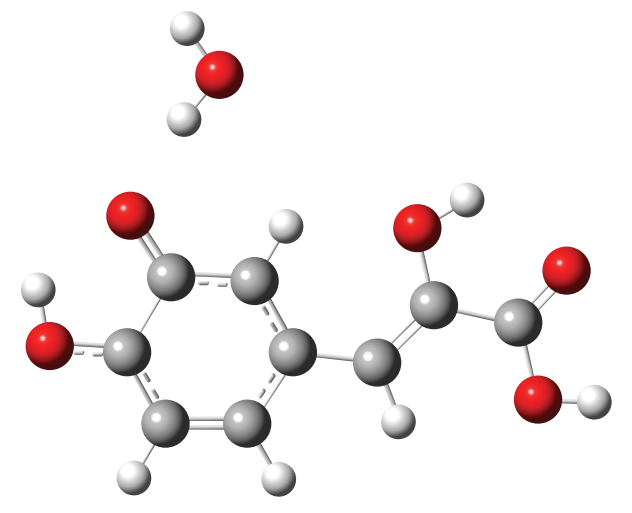

FIGURE 3: Equilibrium geometry of the supermolecule formed after the reaction of 3,4-DHPPA with ${ }^{\circ} \mathrm{OH}$, at B3lyp/6-31+G(d).

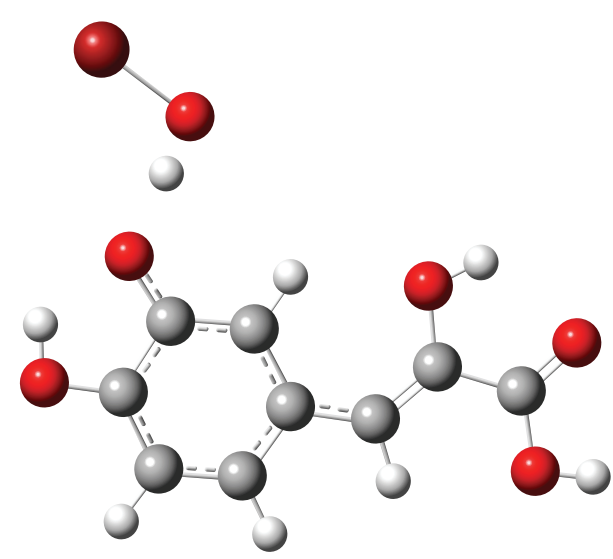

FIGURE 4: Equilibrium geometry of the supermolecule formed after the reaction of 3,4-DHPPA with $\mathrm{OBr}^{-}$, at B3lyp/6-31+G(d).

respectively. Aiming to localize the transition states of these reactions, 3D-relaxed potential energy surfaces (3D-RPES) were performed in the vacuum at the B3LYP/6-31+G* level of theory on the supermolecule obtained, varying the distance $\mathrm{O}_{3}-\mathrm{H}_{3}$ and the angle $\mathrm{C}_{3}-\mathrm{O}_{3}-\mathrm{H}_{3}$, Figures 5 and 6 .

Therefrom, it is noteworthy that the scavenging of hydroxyl radical by 3,4-DHPPA passes through a transition state (TS) while that of $\mathrm{OBr}^{-}$anion is barrierless. Moreover, it is well-known that the reaction of a phenolic acid (PhA) with neutral ROS leads to PCET mechanism while that with negatively charged ROS undergoes a barrierless PT mechanism $[3,12,18,43,44]$. This result may be generalized to PhAs which only have carbon, oxygen, and hydrogen in their skeleton (e.g., Quercetin may be a good candidate) and are involving the same RS or other. Therefore, the scavenging of the hydroxyl radical and the $\mathrm{OBr}^{-}$anion by a $\mathrm{PhA}(\mathrm{ROH})$ can be summarized by the following, respectively:

$$
\begin{gathered}
\mathrm{ROH}+{ }^{\circ} \mathrm{OH} \longrightarrow \mathrm{RO} \cdots \stackrel{\text { ( } \mathrm{TS})}{\mathrm{H}} \cdots \mathrm{OH} \longrightarrow \mathrm{RO}^{\bullet}+\mathrm{H}_{2} \mathrm{O} \\
\mathrm{ROH}+\mathrm{OBr}^{-} \longrightarrow \mathrm{RO}^{-}+\mathrm{HOBr}
\end{gathered}
$$

Natural populations analysis (NPA) and spin densities on reactants, TS, and products, conjointly with singly occupied 


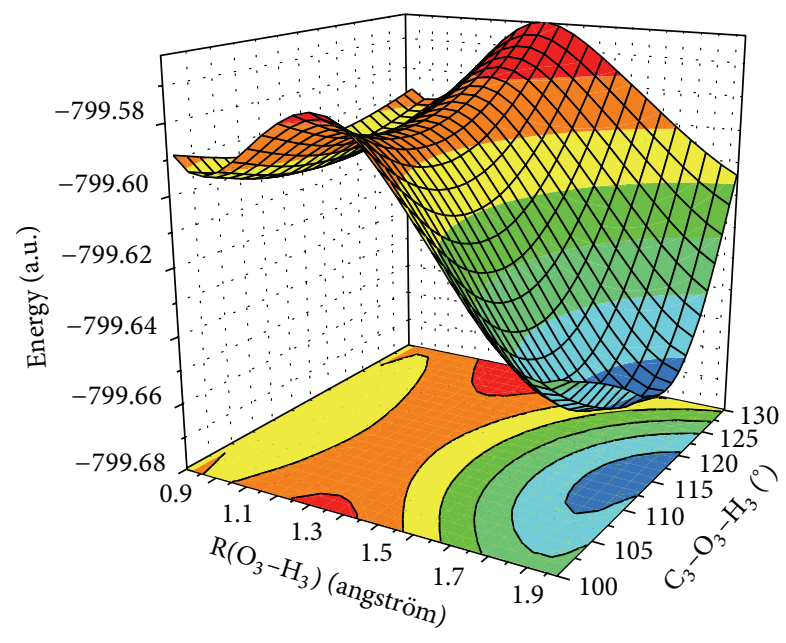

FIGURE 5: 3D-PES of the reaction of 3,4-DHPPA with ${ }^{\circ} \mathrm{OH}$, varying the distance $\mathrm{O}_{3}-\mathrm{H}_{3}$ and the angle $\mathrm{C}_{3}-\mathrm{O}_{3}-\mathrm{H}_{3}$, at B3lyp/6-31+G(d).

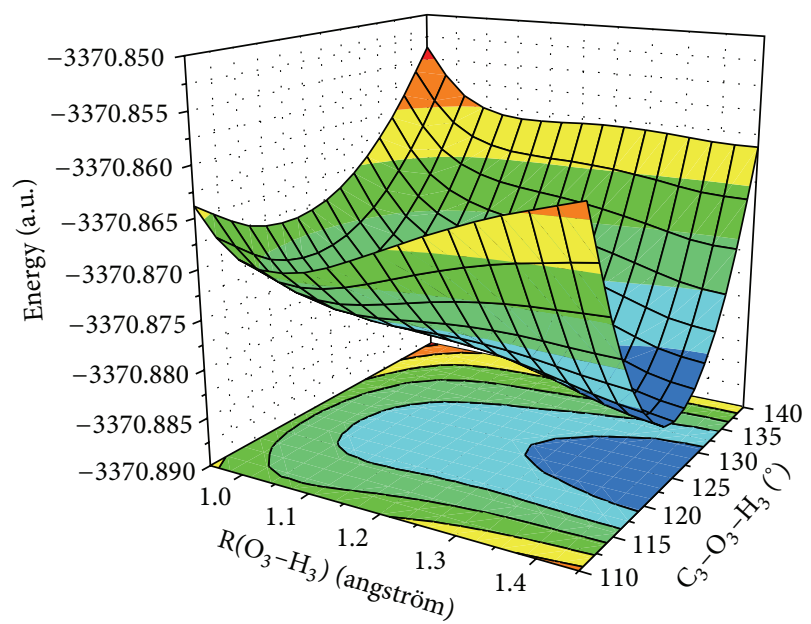

FIGURE 6: 3D-PES of the reaction of 3,4-DHPPA with $\mathrm{OBr}^{-}$, varying the distance $\mathrm{O}_{3}-\mathrm{H}_{3}$ and the angle $\mathrm{C}_{3}-\mathrm{O}_{3}-\mathrm{H}_{3}$, at B3lyp/6-31+G(d).

molecular orbitals (SOMOs) distributions, may provide a good insight in the nature of the particle transferred during the scavenging process.

3.1. Solvation Free Energies of the Electron, Proton, and Hydrogen Atom. Solvation free energies of the electron, proton, and hydrogen atom are, respectively, the free energies change of reactions below [3]:

$$
\begin{gathered}
\operatorname{solvent}_{(\text {solv })}+e_{(\mathrm{g})}^{-} \longrightarrow \text { solvent }_{(\text {solv })}^{-} \\
\operatorname{solvent}_{(\text {solv })}+\mathrm{H}_{(\mathrm{g})}^{+} \longrightarrow \text { solvent- }_{(\text {solv })}^{+} \\
\operatorname{solvent}_{(\text {solv })}+\mathrm{H}_{(\mathrm{g})}^{\cdot} \longrightarrow \text { solvent- }_{(\text {solv })}^{\cdot}
\end{gathered}
$$

where solvent $_{(\text {solv })}$ represents a molecule of solvent in its cavity, $\mathrm{X}_{(\text {solv })}$ means that $\mathrm{X}$ is solvated, and solvent may be one of those used in this work. So, "solv" stands for cyclohexane, benzene, and so forth. Free energies of $\mathrm{X}_{(\text {solv })}$ are obtained from geometry optimizations followed by frequency calculations. As a matter of fact, (20) with water as solvent could be written as

$$
\mathrm{H}_{2} \mathrm{O}_{(\text {water })}+\mathrm{H}_{(\mathrm{g})}^{+} \longrightarrow \mathrm{H}_{2} \mathrm{O}-\mathrm{H}_{(\text {water })}^{+}
$$

It is worthwhile that applying the proposed scheme in the cases of chloroform and dichloromethane is very difficult, since attaching a single electron or proton to the host molecule (chloroform or dichloromethane molecule) leads to the fragmentation of the formed complex. Thus, these solvents are special cases and could be investigated through a more robust scheme. It is also worth mentioning that the solvation free energies of a single electron, proton, and hydrogen atom are not necessary to derive RFEs but they would be very useful to explain the possibility of partial reactions involved in the studied mechanisms. Thus, such parameters help in the thorough investigation of protoncoupled electron transfer in $\mathrm{O}-\mathrm{H}$ bond cleavage.

Gas phase free energy of the electron used here is that obtained by Bartmess: $\Delta_{f} G^{\circ}\left(e^{-}\right)=-3.72 \mathrm{~kJ} / \mathrm{mol}$ [45]. Our calculated gas phase free energy of the proton is $\Delta_{f} G^{\circ}\left(\mathrm{H}^{+}\right)=$ $-26.26 \mathrm{~kJ} / \mathrm{mol}$. Similar results were also proposed recently $[46,47]$. Solvation free energies of $\mathrm{H}$ atom in studied solvents were taken from published solvation free energies and entropies listed in [48-50] and reported in Table 1. In organic solvents, $\Delta_{f} \mathrm{H}_{\text {solv }}\left(\mathrm{H}^{\bullet}\right) \simeq \Delta_{f} \mathrm{H}_{\text {solv }}\left(\mathrm{H}_{2}\right)=(5 \pm$ 1) $\mathrm{kJ} / \mathrm{mol}$ [48-50]. Thus, for organic solvents for which data were unavailable, the value of $5 \mathrm{~kJ} / \mathrm{mol}$ is assumed. Likewise, $\Delta_{f} G_{\text {solv }}\left(\mathrm{H}^{\bullet}\right) \simeq \Delta_{f} G_{\text {solv }}\left(\mathrm{H}_{2}\right) \simeq-20 \mathrm{~kJ} / \mathrm{mol}$. Therefore, this value was retained for the solvents with unavailable experimental values. For water, $\mathrm{H}$-atom hydration free energy reached $27.7 \mathrm{~kJ} / \mathrm{mol}$. Besides, since the only available experimental values for solvation free energies of proton and electron were their hydration free energies $\left(\Delta_{f} G_{\text {hydr }}^{\circ}\left(\mathrm{H}^{+}\right)=\right.$ $\left.-1104.5 \pm 0.3 \mathrm{~kJ} / \mathrm{mol}[51], \Delta_{f} G_{\text {hydr }}^{\circ}\left(e^{-}\right)=-156.8 \mathrm{~kJ} / \mathrm{mol}[52]\right)$, solvation free energies of these particles in different solvents used here were computed at IEF-PCM/B3LYP/6-311++G ${ }^{* *}$ level of theory. In comparison to experimental results, the relative errors on calculated free energies changes for hydrated proton and electron are $9.8 \%$ and $36.8 \%$, respectively. The first error may be acceptable, while the last one is too high. This could mainly be due to the fact that, in our explicit solvation treatment, the first solvation shell is not completely considered. The more accurate scheme should consider at least the first solvation shell completely. This could be undertaken through a thorough investigation of the clustering of protonated, hydrogenated, or electronized solvent. Such a task is beyond the scope of this work and it is devoted to the forthcoming works. For the moment, we assumed that these relative errors are constant in all media. Thus, all calculated $\Delta_{f} G_{\text {solv }}^{\circ}\left(\mathrm{H}^{\circ}\right)$ and $\Delta_{f} G_{\text {solv }}^{\circ}\left(e^{-}\right)$were shifted by an amount of the percentage error and presented in Table 1.

3.2. PCET and PT Mechanisms via SOMOs, NPAs Charges, and Spin Densities Analysis. As a proton and an electron 
TABLE 1: Solvation free energies of hydrogen atom $\left(\Delta_{f} G_{\text {solv }}^{\circ}\left(\mathrm{H}^{\circ}\right)\right)$, proton $\left(\Delta_{f} G_{\text {solv }}^{\circ}\left(\mathrm{H}^{+}\right)\right)$, and electron $\left(\Delta_{f} G_{\text {solv }}^{\circ}\left(e^{-}\right)\right)$in kJ/mol, at 6-311++G $(\mathrm{d}, \mathrm{p})$ in various media. $\varepsilon$ and $\mu$ are, respectively, the dielectric constant and the dipole moment of the considered media.

\begin{tabular}{lccccc}
\hline Solvent & $\varepsilon$ & $\mu$ & $\Delta_{f} G_{\text {solv }}^{\circ}\left(\mathrm{H}^{\circ}\right)$ & $\Delta_{f} G_{\text {solv }}^{\circ}\left(\mathrm{H}^{+}\right)$ & -879.6 \\
\hline Cyclohexane & 2.02 & 0.00 & -20 & -1076.0 & $\Delta_{f} G_{\text {solv }}^{\circ}\left(e^{-}\right)$ \\
Benzene & 2.28 & 0.00 & -20 & -1119.7 & -66.4 \\
Methanol & 32.7 & 1.71 & -20 & -1199.3 & -140.6 \\
DMSO & 46.7 & 3.90 & -20 & -1104.5 & -141.2 \\
Water & 78.4 & 1.80 & 27.7 & -156.8 \\
\hline
\end{tabular}

transfer from a donor to an acceptor site in a molecular system, the charge, spin density, and molecular orbitals (MOs) localized on the donor and acceptors sites change simultaneously. Thus, the calculation of the charge, spin density, and plots of MOs along a reaction coordinate (transferring hydrogen or proton) provides useful insight in understanding the PCET mechanism.

SOMOs distributions on the equilibrium structures resulting from the reaction of 3,4 -DHPPA with ${ }^{\circ} \mathrm{OH}$ in all media show that the radical is entirely delocalised on the phenoxyl moiety (see Figure 7) and could lead to the conclusion that the scavenging of ${ }^{\circ} \mathrm{OH}$ by 3,4 -DHPPA is governed by a hydrogen atom transfer (PCET mechanism) whatever the media.

NPAs and spin densities computations were performed at the same level of computations as that of geometry optimization. Computed NPA charges for products of the reaction illustrated by (17) are neutral, while spin densities are, respectively, 1.00 and 0.00 for the phenoxyl moiety and the water molecule formed, whatever the medium. In addition, the computation of such charges on reactants, products, and transition state of this reaction shows that the transferred particle has a positive charge with no spin associated, Table 2. Moreover, it is worth mentioning that the spin density associated with the phenoxyl moiety at the TS is increasing while that of the hydroxyl radical is decreasing, going from reactants to products. This indicates that the radical transfer is occurring. Moreover, at the TS, the negative NPA charges on $\mathrm{OH}$ increase with the solvent polarity while its spin density decreases with it, until spin density is no longer associated with $\mathrm{OH}$ in polar solvents. Note that, in such solvents, the electron spin density on $\mathrm{OH}$ is quite nil, while it is a unit on the phenoxyl moiety (RO), Table 2. Moreover, the electron charge on $\mathrm{OH}$ is more than the half of elementary electron charge. Thus, while the reaction of (17) is proceeding in polar solvents, the radical character is completely transferred from the free radical $\left({ }^{\circ} \mathrm{OH}\right)$ to the phenoxyl moiety before the proton is transferred from the acid to the free radical. Therefore, in polar solvents, the scavenging of the hydroxyl radical by DHPPA is stepwise and the electron is transferred first. In the vacuum and nonpolar solvents, the electron spin densities on $\mathrm{OH}$ and $\mathrm{RO}$ are about 0.2 and 0.8 , respectively. In addition, the electron charge on $\mathrm{OH}$ is less than the unit. Thus, the located TS for the reaction of (17) is a mixture of ET and PT characters. Therefore, a clear distinction of ET-PT, PT-ET, and CPCET mechanisms

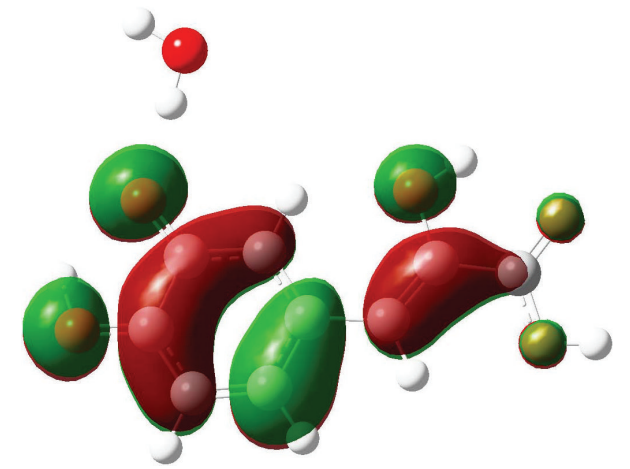

FIGURE 7: SOMO distribution on the equilibrium structure of the supermolecule resulting from the reaction of 3,4-DHPPA with ${ }^{\circ} \mathrm{OH}$ radical: a B3lyp/6-31+G(d). SOMO is entirely delocalized on the phenoxyl moiety, suggesting hydrogen atom transform from the acid to the radical.

TABLE 2: NPA charges $(e)$ and spin densities carried by elements of the reaction at the transition state. The superscripts 3 and 4 on DHPPA indicate that the hydrogen atom concerned by the abstraction is $\mathrm{H}_{3}$ and $\mathrm{H}_{4}$, respectively. The symbol $\mathrm{X}$ denotes the unknown transferred particle.

\begin{tabular}{cccccccc}
\hline & \multirow{2}{*}{ Media } & \multicolumn{3}{c}{ NPA charges } & \multicolumn{3}{c}{ Spin densities } \\
& & RO & X & OH & RO & X & OH \\
\hline \multirow{2}{*}{ Vacuum } & -0.02 & 0.52 & -0.50 & 0.73 & 0.00 & 0.27 \\
& Cyclohexane & 0.11 & 0.52 & -0.63 & 0.82 & 0.00 & 0.18 \\
& Benzene & 0.10 & 0.53 & -0.63 & 0.83 & 0.00 & 0.17 \\
& Methanol & 0.26 & 0.52 & -0.78 & 0.96 & 0.00 & 0.04 \\
& Water & 0.25 & 0.52 & -0.79 & 0.98 & 0.00 & 0.02 \\
\hline \multirow{2}{*}{$\mathrm{DHPPA}_{4} 4$} & Vacuum & -0.02 & 0.52 & -0.50 & 0.76 & 0.00 & 0.24 \\
& Cyclohexane & - & - & - & - & - & - \\
& Benzene & 0.12 & 0.53 & -0.65 & 0.89 & 0.00 & 0.11 \\
& Methanol & 0.28 & 0.55 & -0.83 & 0.99 & 0.00 & 0.01 \\
& Water & 0.29 & 0.55 & -0.84 & 0.99 & 0.00 & 0.01 \\
\hline
\end{tabular}

for such a reaction is not ensured using NPA charges and spin densities at the TS. Parameters based on reaction free energies and activation energies could be good indicators for such a target. 


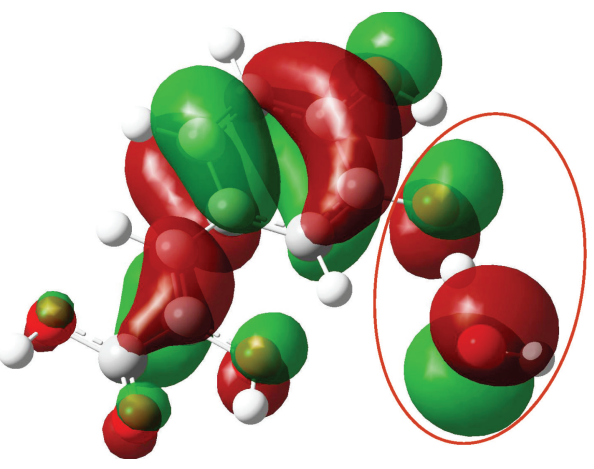

FIGURE 8: SOMO distribution on a transition state structure of the reaction of D-DHPPA with ${ }^{\circ} \mathrm{OH}$ radical in the vacuum.

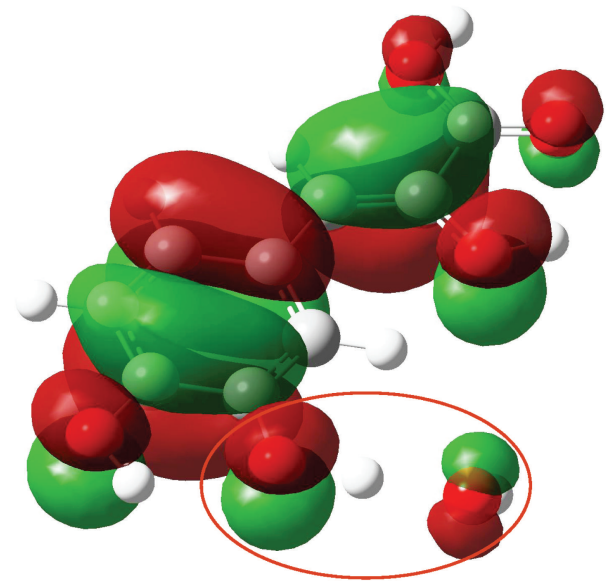

FIGURE 9: SOMO distribution on a transition state structure of the reaction of D-DHPPA with ${ }^{\circ} \mathrm{OH}$ radical in water.

Moreover, we plotted the SOMOs at the TS of this reaction (17) in different solvents. These SOMOs are delocalised entirely on the supermolecule, Figures 8 and 9. These SOMOs show that the electron and proton transfer from different orbitals on the donor to different orbitals on the acceptor. The same spin density distribution was noted in other solvents (see Figure S1 in the Supplementary Material available online at http://dx.doi.org/10.1155/2015/835707).

Thus, based on the works of Mayer et al. [53] and Hammes-Schiffer and Iordanova [54], these SOMOs distributions allow us to conclude that the scavenging of hydroxyl radical by D-DHPPA is governed by a CPCET mechanism, whatever the medium. Hence, in contrast to the results published before (the scavenging of hydroxyl radical by DHPPA is governed by CPCET in nonpolar solvents and PTET mechanisms in polar solvents [3]), PT-ET mechanism may no longer be favored in polar solvents if coreactive species are taken into account.

Regarding the reaction illustrated by (18), for which there is neither radical nor transition state involved, NPA calculations show that the resulting phenoxyl moiety bears a negative charge while the $\mathrm{HOBr}$ formed is neutral. This reveals a proton transfer and confirms the well-known result concerning the nature of the scavenging of a negatively charged species by a PhA.

3.3. Thermodynamic Investigation of PCET and PT Mechanisms. Reaction free energies relevant to different pathways related to $\mathrm{PT}-\mathrm{ET}$, ET-PT, CPCET, and PT mechanisms are based on coreaction free energies (Table 3 ) and abstraction free energies of ${ }^{\bullet} \mathrm{H}, \mathrm{H}^{+}$, and $e^{-}$(Table 4). Coreaction free energies are assumed to be the free energies of the reactions involving coreactive species and abstracted or transferred particle $\left({ }^{\bullet} \mathrm{H}, \mathrm{H}^{+}\right.$, and $\left.e^{-}\right)$. It is noteworthy that RFE-CPCET may not be used in concert with RFE-ET(1) and RFE-PT(2) to derive the preferred mechanism (PCET? PT-ET? or ET-PT?) in a given medium. In fact, the former characterizes the onestep $\mathrm{H}$-atom transfer, while the other characterizes one step in a stepwise $\mathrm{H}$-atom transfer. Thus, a reliable thermodynamic way to derive the preferred mechanism in a given medium is to compare RFE-ET(1), RFE-PT(2), and the activation barrier $\left(\Delta G^{\neq}\right)$of the CPCET mechanism.

3.3.1. CPCET Mechanism. Previously, studies on the CPCET mechanism at $3-\mathrm{OH}, 4-\mathrm{OH}$, and $8-\mathrm{OH}$ positions in 3,4DHPPA have shown that $\mathrm{BDE}_{3}<\mathrm{BDE}_{8}<\mathrm{BDE}_{4}$ in the vacuum and nonpolar solvents [3]. This order changes in polar solvents and becomes $\mathrm{BDE}_{8}<\mathrm{BDE}_{4}<\mathrm{BDE}_{3}$. These behaviors were also obtained when coreactive species were taken into account, Figure 10 (or Table S1). The justification of such a result was based on the relative strengths of hydrogen bonds $\mathrm{O}_{4} \mathrm{H}_{4} \cdots \mathrm{O}_{8}, \mathrm{O}_{8} \mathrm{H}_{8} \cdots \mathrm{O}_{9}$ and $\mathrm{C}_{2} \mathrm{H}_{2} \cdots \mathrm{O}_{8}$ in 3,4DHPPA. Details were discussed in our previous manuscript [3]. Thus, it may be sufficient to study the CPCET mechanism using BDFEs or BDEs rather than RFEs, in order to derive the relative ability to abstract the $\mathrm{H}$-atom through the abstraction sites of the PhA. Note that the negative values obtained for RFE-CPCET in all media are the confirmation that the transfer of the $\mathrm{H}$-atom from the acid to the hydroxyl radical is always possible in such media.

3.3.2. PT-ET Mechanisms. Assume that the reaction of 3,4DHPPA with ${ }^{\circ} \mathrm{OH}$ radical processes through a PT-ET mechanism leads to the following reaction:

$$
\mathrm{ROH}+{ }^{\bullet} \mathrm{OH} \longrightarrow \mathrm{RO}^{-}+\mathrm{H}_{2} \mathrm{O}^{+} \longrightarrow \mathrm{RO}^{\bullet}+\mathrm{H}_{2} \mathrm{O}
$$

All the RFE-PT(2)(OH) values obtained are positive, Table $\mathrm{S} 1$. Thus, such a reaction is always thermodynamically unfavorable, especially in the vacuum. This may be explained by the fact that the fragmentation of a neutral molecule into a charged species is more difficult in the vacuum than in solvent, since solvent will make the molecule more polar and thereafter easy to be fragmented in charged species. Moreover, the reaction involves the reaction of ${ }^{\circ} \mathrm{OH}$ with a proton $\mathrm{H}^{+}$. This reaction is usually less favorable, especially in solvent. Indeed, the free radical ${ }^{\circ} \mathrm{OH}$ is neutral and polar, so it cannot interact easily with a charged and nonpolar molecular system. This physical insight is confirmed through RFEs of the reactions ${ }^{\bullet} \mathrm{OH}+\mathrm{H}^{+} \rightarrow \mathrm{H}_{2} \mathrm{O}^{+}$, Table 3 . 
TABLE 3: Free energies of reactions involving coreactive species and abstracted or transferred particle $\left({ }^{\circ} \mathrm{H}, \mathrm{H}^{+}\right.$, and $\left.e^{-}\right)$in $\mathrm{kJ} / \mathrm{mol}$ at $\mathrm{B} 3 \mathrm{LYP} / 6-$ $31+G(d)$.

\begin{tabular}{lcccc}
\hline Media & $\cdot \mathrm{OH}+e^{-} \rightarrow \mathrm{OH}^{-}$ & $\cdot \mathrm{OH}+{ }^{\bullet} \mathrm{H} \rightarrow \mathrm{H}_{2} \mathrm{O}$ & $\mathrm{OBH}^{-}+\mathrm{H}^{+} \rightarrow \mathrm{H}_{2} \mathrm{O}^{+} \rightarrow \mathrm{HOBr}^{+}$ \\
\hline Vacuum & -160.3 & -436.0 & -544.1 & -1438.1 \\
Cyclohexane & -329.0 & -418.8 & 35.4 & -536.3 \\
Benzene & -332.2 & -419.1 & 212.6 & -327.4 \\
Methanol & -397.7 & -422.0 & 90.1 & -180.3 \\
DMSO & -400.1 & -422.1 & 165.8 & -98.6 \\
Water & -387.3 & -469.8 & 67.3 & -191.3 \\
\hline
\end{tabular}

TABLE 4: Abstraction free energies of electron, proton, and hydrogen atom from a phenolic acid ROH in $\mathrm{kJ} / \mathrm{mol}$.

\begin{tabular}{|c|c|c|c|c|}
\hline & Media & $\mathrm{ROH} \rightarrow \mathrm{ROH}^{+}+e^{-}$ & $\mathrm{ROH} \rightarrow \mathrm{RO}^{-}+\mathrm{H}^{+}$ & $\mathrm{ROH} \rightarrow \mathrm{RO}^{\bullet}+{ }^{\circ} \mathrm{H}$ \\
\hline \multirow{6}{*}{$\mathrm{DHPPA}_{3}$} & Vacuum & 699.4 & 1339.2 & 270.0 \\
\hline & Cyclohexane & 604.8 & 488.3 & 254.4 \\
\hline & Benzene & 581.9 & 283.5 & 254.8 \\
\hline & Methanol & 428.7 & 172.9 & 263.6 \\
\hline & DMSO & 428.0 & 91.9 & 263.7 \\
\hline & Water & 408.1 & 185.8 & 310.8 \\
\hline \multirow{6}{*}{$\mathrm{DHPPA}_{4}$} & Vacuum & 699.4 & 1355.3 & 288.4 \\
\hline & Cyclohexane & 604.8 & 500.3 & 264.0 \\
\hline & Benzene & 581.9 & 295.4 & 263.2 \\
\hline & Methanol & 428.7 & 174.4 & 256.3 \\
\hline & DMSO & 428.0 & 92.9 & 256.1 \\
\hline & Water & 408.1 & 186.5 & 304.6 \\
\hline \multirow{6}{*}{$\mathrm{DHPPA}_{8}$} & Vacuum & 699.40 & 1363.5 & 280.5 \\
\hline & Cyclohexane & 604.78 & 506.9 & 257.3 \\
\hline & Benzene & 581.87 & 301.6 & 256.8 \\
\hline & Methanol & 428.71 & 174.9 & 252.7 \\
\hline & DMSO & 428.04 & 94.9 & 253.0 \\
\hline & Water & 408.1 & 187.4 & 298.5 \\
\hline
\end{tabular}

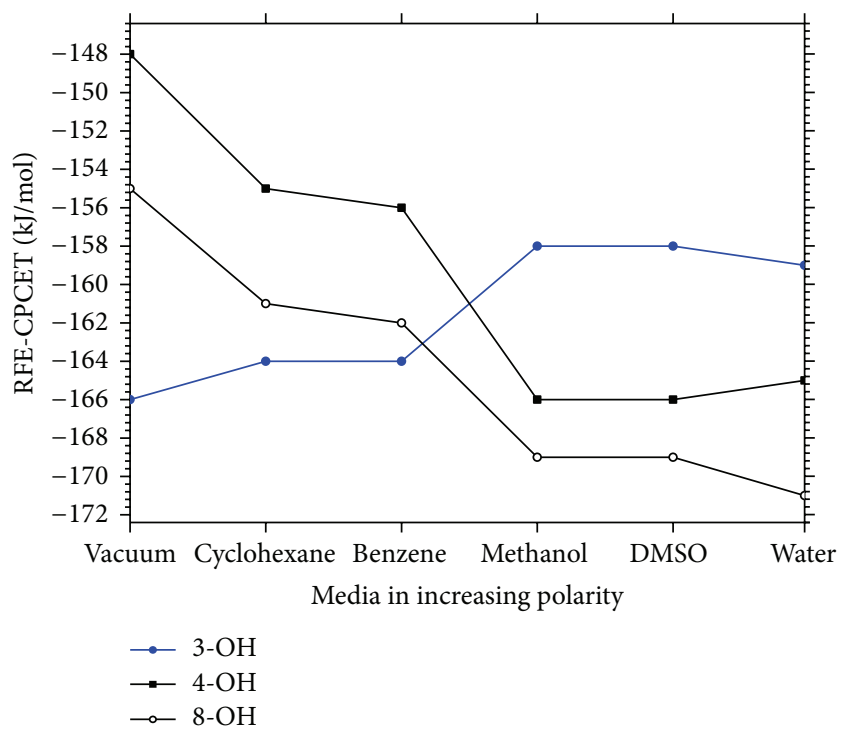

FIGURE 10: Reaction free energies for the reaction $\mathrm{ROH}+{ }^{\circ} \mathrm{OH} \rightarrow$ $\mathrm{RO}^{\bullet}+\mathrm{H}_{2} \mathrm{O}$, in different media. $\mathrm{ROH}$ stands for 3,4-DHPPA, while $\mathrm{RO}^{\bullet}$ stands for its phenoxyl moiety.
Furthermore, the scavenging of the ${ }^{\bullet} \mathrm{OH}$ radical by 3,4 DHPPA through the PT-ET mechanism is done in all abstraction sites $(3-\mathrm{OH}, 4-\mathrm{OH}$, and $8-\mathrm{OH})$ with the same ability in polar solvents, Figure 11. In the vacuum and nonpolar solvents, the $3-\mathrm{OH}$ site is slightly preferred to the $4-\mathrm{OH}$ one, which in turn is slightly preferred to the $8-\mathrm{OH}$ site. In addition, this mechanism (PT-ET) is more favorable in solvent than in the vacuum, and the more the solvent polarity, the more favorable the mechanism, Figure 12. This may be explained through its dipole moment by the fact that solvent eases charge separation and, the more the solvent polarity, the easier the charge separation. These results are consistent with those found without taking into account the coreactive species [3].

By comparing the activation barrier $\Delta G^{\neq}$of the CPCET mechanism with RFE-PT(2), one can predict that, even kinetically, the CPCET mechanism would be preferred to the PT-ET mechanism in all media, Figure 12. The justification of this statement could also be performed by a kinetic investigation of the PT-ET mechanism which is beyond the scope of this work. 


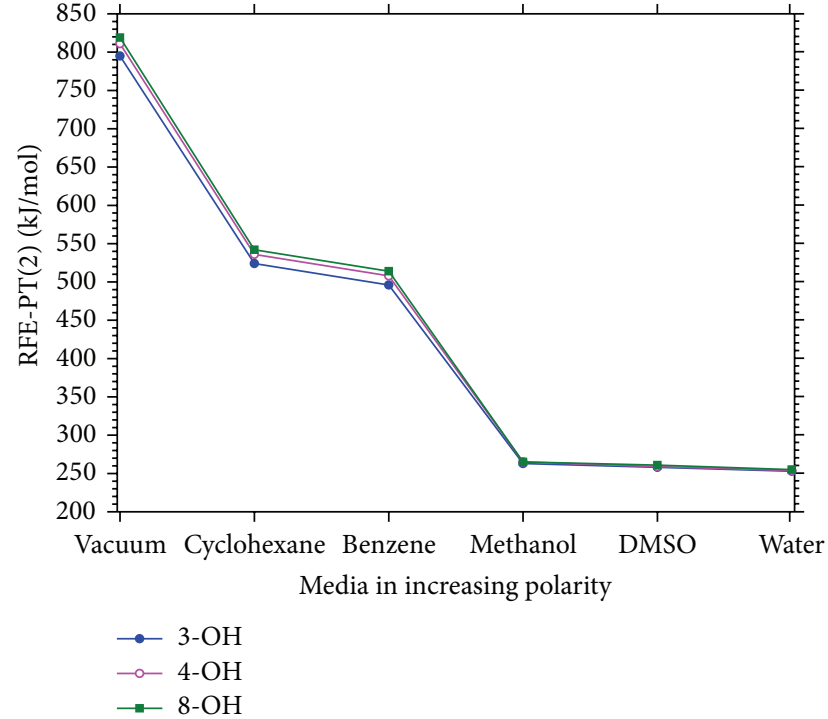

FIGURE 11: Reaction free energies evaluating the possibility of the mechanism PT-ET in the reaction of 3,4-DHPPA with ${ }^{\circ} \mathrm{OH}$ in different media. These RFEs are calculated as the sum of partial reaction free energies of the following reactions: $\mathrm{ROH} \rightarrow \mathrm{RO}^{-}+\mathrm{H}^{+}$, $\mathrm{OH}+\mathrm{H}^{+} \rightarrow \mathrm{H}_{2} \mathrm{O}^{+}$. $\mathrm{ROH}$ stands for 3,4-DHPPA, while $\mathrm{RO}^{-}$stands for its deprotonated structure. $3-\mathrm{OH}, 4-\mathrm{OH}$, and $8-\mathrm{OH}$ denote the cleavage sites related, respectively, to the protons $\mathrm{H}_{3}^{+}, \mathrm{H}_{4}^{+}$, and $\mathrm{H}_{8}^{+}$of 3,4-DHPPA.

3.3.3. ET-PT Mechanism. Assume that the reaction of 3,4DHPPA with ${ }^{\circ} \mathrm{OH}$ radical processes through a ET-PT mechanism leads to the following reaction:

$$
\mathrm{ROH}+{ }^{\bullet} \mathrm{OH} \longrightarrow \mathrm{ROH}^{+}+\mathrm{OH}^{-} \longrightarrow \mathrm{RO}^{\bullet}+\mathrm{H}_{2} \mathrm{O}
$$

This reaction is thermodynamically more favorable in solvent than in the vacuum, and the more the solvent polarity, the more favorable the mechanism, Figure 12. These results are consistent with the earlier explanation relevant to the easiness of the solvent to separate charged species. They are also consistent with those found without taking into account the coreactive species [3].

By comparing the activation barrier $\Delta G^{\neq}$of the CPCET mechanism with RFE-ET(1), one can predict that, even kinetically, the CPCET mechanism would be preferred in the vacuum and nonpolar solvents compared to ET-PT mechanism which in turn would be preferred in polar solvents, Figure 12. Furthermore, in polar solvents, ET-PT mechanism competes strongly with CPCET mechanism, Figure 12. The justification of these kinetic viewpoints could also be performed by a kinetic investigation of the ET-PT mechanism which is beyond the scope of this work.

Therefore, during the scavenging of the hydroxyl radical by DHPPA, the transfer of the hydrogen atom is thermodynamically concerted in all media and favoring by this way the CPCET mechanism in all media. However, in kinetic viewpoint, this mechanism could compete strongly with the ET-PT mechanism so that the latter could slightly dominate. These results which corroborate the results obtained through the SOMOs distribution on the TS structures are also in

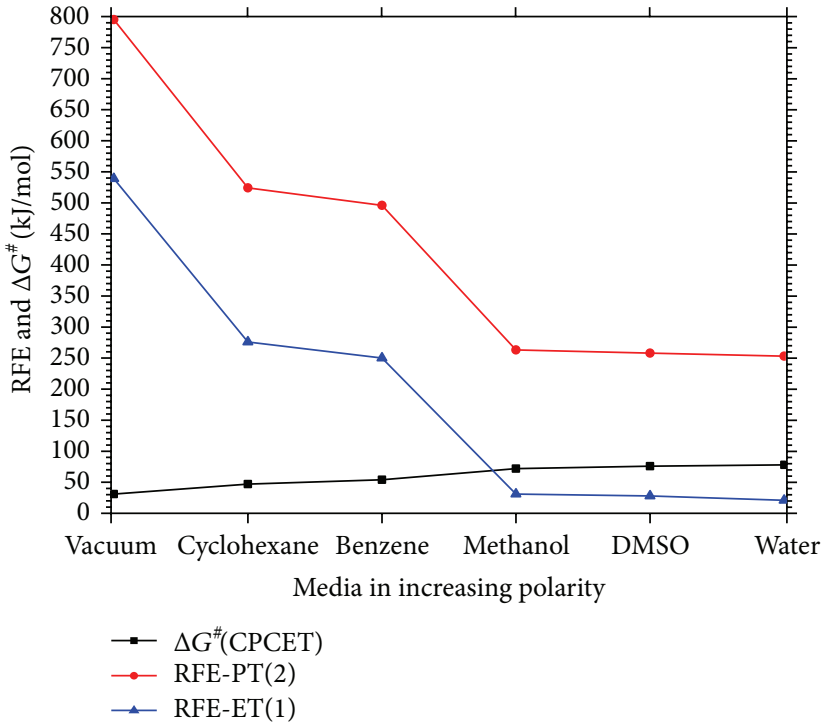

FIGURE 12: Reaction free energies and activation barriers evaluating, respectively, the possibility of the mechanisms PT-ET, ET-PT, and CPCET, in the reaction of 3,4-DHPPA with ${ }^{\circ} \mathrm{OH}$ in different media. For CPCET, the reaction involved is $\mathrm{ROH}+{ }^{\circ} \mathrm{OH} \rightarrow \mathrm{RO}^{\circ}+\mathrm{H}_{2} \mathrm{O}$. For PT-ET, the partial reactions involved are $\mathrm{ROH} \rightarrow \mathrm{RO}^{-}+\mathrm{H}^{+}$, $\mathrm{OH}+\mathrm{H}^{+} \rightarrow \mathrm{H}_{2} \mathrm{O}^{+}$. For ET-PT, the partial reactions involved are $\mathrm{ROH} \rightarrow \mathrm{ROH}^{+}+e^{-}, \cdot \mathrm{OH}+e^{-} \rightarrow \mathrm{OH}^{-}$. $\mathrm{ROH}$ stands for 3,4DHPPA.

agreement with those derived in polar solvents using NPA charges and electron spin densities at TS. They also bring a clear decision on the preferred mechanism in the vacuum and nonpolar solvents. It is noteworthy that the obtained results are consistent in the vacuum and nonpolar solvents with previous results $[3,5,6,9]$ which do not involve coreactive species. The differences were noted in polar solvents. This difference could be explained by the fact that the approach free of coreactives involvement is unable to give a kinetic insight of such a reaction.

3.3.4. PT Mechanism. PT mechanism is involved in the scavenging of the anion $\mathrm{OBr}^{-}$by 3,4-DHPPA. This mechanism is less favorable in solvents than in the vacuum, and the more the solvent polarity increases, the less the mechanism is favored, Figure 13 (or Table S1). This result may also be explained by the easiness to separate charged species in the vacuum compared to in solvents, especially in polar solvents.

3.4. Kinetic Study of the PCET Mechanism. To complete the above thermodynamic description, kinetic investigation of the PCET mechanism (17) was undertaken in various media. The media retained were vacuum, cyclohexane, benzene, methanol, and water. The basis set superposition error (BSSE) was computed for the transition state structure at the G3B3 level of computation in the vacuum. For the TS structures related to the $\mathrm{H}_{3}$-atom, $\mathrm{H}_{4}$-atom, and $\mathrm{H}_{8}$-atom abstractions in the vacuum, the obtained values were 12.6, 12.4, and $12.3 \mathrm{~kJ} / \mathrm{mol}$, respectively. The same values were used in solvents, since up to now there are no possible counterpoise 


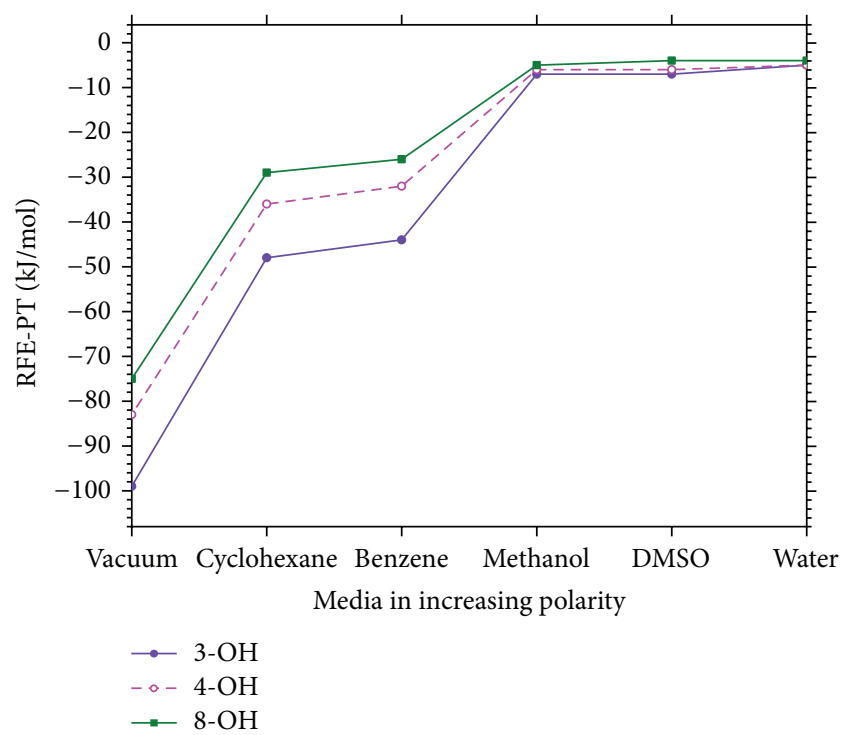

FIGURE 13: Reaction free energies related to proton transfer (PT) involving the scavenging of the anion $\mathrm{OBr}^{-}$by 3,4-DHPPA in different media. RFE-PT is calculated as the sum of partial RFEs of the reactions $\mathrm{ROH} \rightarrow \mathrm{RO}^{-}+\mathrm{H}^{+}$and $\mathrm{OBr}^{-}+\mathrm{H}^{+} \rightarrow \mathrm{HOBr}$.

computations in solvent. Rate constants were computed at $T=298.15 \mathrm{~K}$, using TST/ST and TST/ZCT methods. The TST/ST method is the traditional TST method with $\kappa_{\text {ST }}^{<}$ for the transmission coefficient and is implemented in our homemade Fortran program. The computed activation free energies $\left(\Delta G^{\neq}\right)$were achieved including zero-point energies.

The rate constants obtained from both described methods are quite consistent (see Figure 14) and lead to the same information that rate constants decrease as the solvent polarity increases. The result is also consistent with the increasing distance $d\left(\mathrm{O}_{n} \cdots \mathrm{H}_{n}\right)$ with the solvent polarity, $n=3,4$. It is also in good agreement with the decreasing frequency $v\left(\mathrm{O}_{n} \cdots \mathrm{H}_{n}\right)$. Indeed, the longer distance matches a lower frequency, since a longer distance corresponds to a weaker bond. The discrepancy observed in the vacuum between both methods of rate constants calculation could be due to the large Wigner tunnelling effects in such a medium. This shows that the tunnelling effects on hydrogen atom transfer could be more important in the vacuum than in a solvent. Indeed, the TS vibrational frequency in the vacuum is $-2429 \mathrm{~cm}^{-1}$, while in a solvent the value is about $-1500 \mathrm{~cm}^{-1}$, which is lower than the former.

This result is consistent with the increasing activation barrier with solvent polarity (Table 5), suggesting that the higher the solvent polarity, the slower the CPCET mechanism. This result is not surprising, since it is shown early that solvent makes the one-step H-atom transfer harder.

Moreover, whatever the medium, the hydrogen atom $\mathrm{H}_{3}$ is transferred more rapidly than the hydrogen ato $\mathrm{H}_{4}$, since the related rate constant of $\mathrm{H}_{3}$ transfer is higher than that related to $\mathrm{H}_{4}$ transfer in a given medium, Table 5. In addition, the activation free energy and rate constants using TST/ST and TST/ZCT methods for the $\mathrm{O}_{8}-\mathrm{H}_{8}$ bond cleavage in

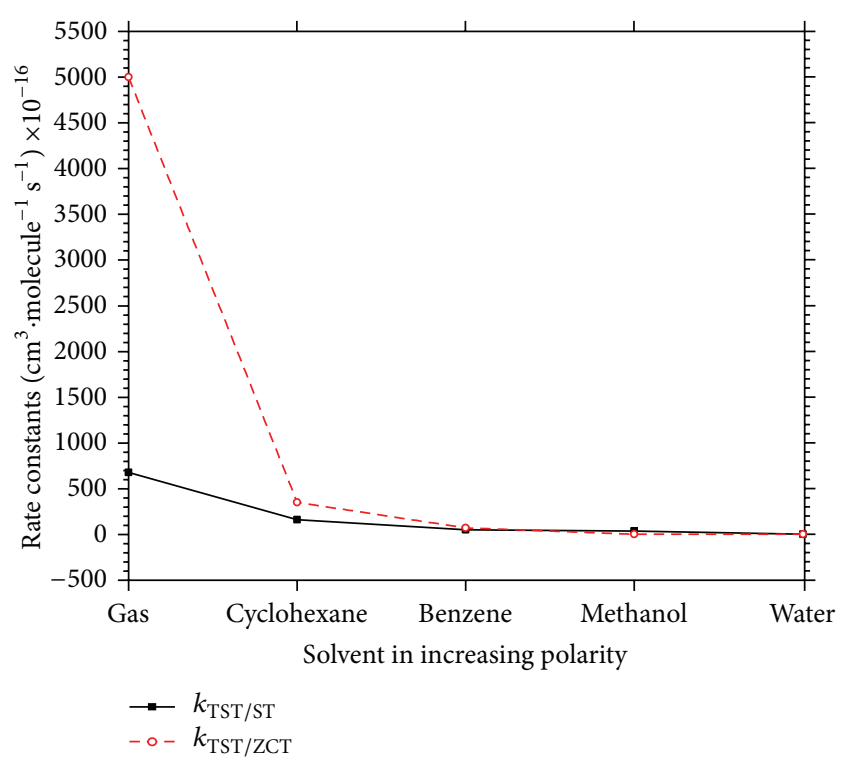

FIGURE 14: Rate constants for the scavenging of the free radical ${ }^{\circ} \mathrm{OH}$ by the 3,4-DHPPA, computed using TST/ST and TST/ZCT methods in different media. The attacked site considered is $\mathrm{O}_{3}-\mathrm{H}_{3}$.

the vacuum are $70.1 \mathrm{~kJ} / \mathrm{mol}, 1.3 \times 10^{-17} \mathrm{~cm}^{3} \cdot$ molecule ${ }^{-1} \cdot \mathrm{s}^{-1}$, and $4.2 \times 10^{-16} \mathrm{~cm}^{3} \cdot \mathrm{molecule}^{-1} \cdot \mathrm{s}^{-1}$, respectively. Thus, the rate constant related to $\mathrm{H}_{4}$ transfer is higher than that related to $\mathrm{H}_{8}$ in the vacuum.

\section{Conclusion}

In this work, we investigated the reaction of 3,4-dihydroxyphenylpyruvic acid (3,4-DHPPA) with hydroxyl radical $\left({ }^{\circ} \mathrm{OH}\right)$ and bromite ion $\left(\mathrm{OBr}^{-}\right)$in various media (vacuum, benzene, cyclohexane, methanol, DMSO, and water). The goal of this work was to distinguish the concerted protoncoupled electron transfer (CPCET) from sequential one, as well as proton transfer-electron transfer (PT-ET) from electron transfer-proton transfer (ET-PT) in the O-H bond cleavage reactions in various media.

As a result, we found that the scavenging of hydroxyl radical by 3,4-DHPPA is thermodynamically governed by a one-step hydrogen atom transfer (CPCET) from the acid to the radical in all media. In kinetic viewpoint, CPCET still dominates in the vacuum and in nonpolar solvents, but in polar solvents it could compete strongly with the ETPT mechanism so that the latter could slightly dominate. In kinetic viewpoint, this mechanism could compete strongly with the ET-PT mechanism so that the latter could slightly dominate. In addition, the rate constant of the CPCET mechanism decreases going from the vacuum to the solvent, and the higher the solvent polarity is, the lower the rate constant is. Thus, in the vacuum and nonpolar solvents, results that take into account the coreactive species are similar to those that do not. The differences were noted in polar solvents. For the reaction $\mathrm{ROH}+\mathrm{OBr}^{-} \rightarrow \mathrm{RO}^{-}+\mathrm{HOBr}$, one can retain that, being more favored in the vacuum than in 
TABLE 5: Parameters characterizing the $\mathrm{O}-\mathrm{H}$ bond cleavage in selected media. $d\left(\mathrm{O}_{n} \cdots \mathrm{H}_{n}\right)$ in $\AA$ is the distance from the oxygen $\mathrm{O}_{n}$ of the phenoxyl moiety to the moved hydrogen atom $\mathrm{H}_{n} \cdot \nu\left(\mathrm{O}_{n} \cdots \mathrm{H}_{n}\right)$ in $\mathrm{cm}^{-1}$ is the corresponding frequency associated with the movement of this hydrogen atom, while $k_{\mathrm{TST} / \mathrm{ST}}$ and $k_{\mathrm{TST} / \mathrm{ZCT}}$ in $\mathrm{cm}^{3} \cdot$ molecule $^{-1} \cdot \mathrm{s}^{-1}$ are the associated rate constants using, respectively, TST/ST and TST/ZCT methods. $\Delta G^{\neq}$in $\mathrm{kJ} / \mathrm{mol}$ is the activation free energy, and $n=3,4$. NA stands for not applicable.

\begin{tabular}{|c|c|c|c|c|c|}
\hline Media & $d$ & $v$ & $\Delta G^{\neq}$ & $k_{\mathrm{TST} / \mathrm{ST}}$ & $k_{\mathrm{TST} / \mathrm{ZCT}}$ \\
\hline \multicolumn{6}{|c|}{ Related to the $\mathrm{O}_{3}-\mathrm{H}_{3}$ attacked site } \\
\hline Vacuum & 0.969 & 3769 & 31.2 & $6.8 \times 10^{-14}$ & $5.0 \times 10^{-13}$ \\
\hline Cyclohexane & 0.975 & 3645 & 47.0 & $1.6 \times 10^{-14}$ & $3.5 \times 10^{-14}$ \\
\hline Benzene & 0.976 & 3625 & 53.8 & $5.1 \times 10^{-15}$ & $7.1 \times 10^{-15}$ \\
\hline Methanol & 0.990 & 3331 & 71.8 & $3.6 \times 10^{-15}$ & $2.5 \times 10^{-16}$ \\
\hline Water & 0.991 & 3326 & 78.3 & $4.8 \times 10^{-16}$ & $1.6 \times 10^{-16}$ \\
\hline \multicolumn{6}{|c|}{ Related to the $\mathrm{O}_{4}-\mathrm{H}_{4}$ attacked site } \\
\hline Vacuum & 0.964 & 3710 & 68.1 & $1.9 \times 10^{-17}$ & $6.2 \times 10^{-16}$ \\
\hline Cyclohexane & 0.975 & 3661 & - & - & - \\
\hline Benzene & 0.976 & 3652 & 78.6 & $2.9 \times 10^{-18}$ & NA \\
\hline Methanol & 0.983 & 3493 & 93.3 & $1.7 \times 10^{-19}$ & $6.1 \times 10^{-18}$ \\
\hline Water & 0.985 & 3457 & 90.9 & - & - \\
\hline
\end{tabular}

solvent, it proceeds through a proton transfer from the acid to the reactive oxygen species, and the more the solvent polarity increases, the less the mechanism is favored.

Therefore, studying the $\mathrm{O}-\mathrm{H}$ bond cleavage subsequent to the scavenging of reactive oxygen species by a phenolic acid through simple thermodynamic parameters such as bond dissociation free energy, proton affinity, or ionization potential is only satisfactory in the thermodynamic viewpoint. Reaction free energy (RFE) parameters could be reliable to have both thermodynamic and kinetic viewpoints in such a reaction. Moreover, although the RFE may not be necessary for the concerted PCET mechanism, it may be very useful and may be a deciding factor for the investigations of sequential proton-coupled electron transfer and proton transfer mechanisms.

\section{Conflict of Interests}

The authors declare that there is no conflict of interests regarding the publication of this paper.

\section{Acknowledgments}

Authors thank the Abdus Salam ICTP for their financial support to this work through the OEA-NET 45 Project. They are also grateful to Professor Donald G. Truhlar, Department of Chemistry, University of Minnesota, for the license access to POLYRATE 2010A suite of codes. J. J. Fifen thanks the Center for High Performance Computing (CHPC) in South Africa for granting him access to their clusters and computational resources. He also thanks Professor Emeritus Zohra Ben Lakhdar (University of Tunis El Manar, Tunisia) and Professor Souad Chekir Lahmar (University of Carthage, Tunisia) for their warm hospitality in Tunisia where this work was achieved.

\section{References}

[1] R. I. Cukier and D. G. Nocera, "Proton-coupled electron transfer," Annual Review of Physical Chemistry, vol. 49, no. 1, pp. 337-369, 1998.

[2] M. H. V. Huynh and T. J. Meyer, "Proton-coupled electron transfer," Chemical Reviews, vol. 107, no. 11, pp. 5004-5064, 2007.

[3] J. J. Fifen, M. Nsangou, Z. Dhaouadi, O. Motapon, and N. Jaidane, "Solvent effects on the antioxidant activity of 3,4dihydroxyphenylpyruvic acid: DFT and TD-DFT studies," Computational and Theoretical Chemistry, vol. 966, no. 1-3, pp. 232-243, 2011.

[4] K. Senthil kumar and R. Kumaresan, "A DFT Study on the structural, electronic properties and radical scavenging mechanisms of calycosin, glycitein, pratensein and prunetin," Computational and Theoretical Chemistry, vol. 985, pp. 14-22, 2012.

[5] M. Najafi, M. Zahedi, and E. Klein, "DFT/B3LYP study of the solvent effect on the reaction enthalpies of homolytic and heterolytic $\mathrm{O}-\mathrm{H}$ bond cleavage in mono-substituted chromans," Computational and Theoretical Chemistry, vol. 978, no. 1-3, pp. 16-28, 2011.

[6] M. Najafi, K. H. Mood, M. Zahedi, and E. Klein, "DFT/B3LYP study of the substituent effect on the reaction enthalpies of the individual steps of single electron transfer-proton transfer and sequential proton loss electron transfer mechanisms of chroman derivatives antioxidant action," Computational and Theoretical Chemistry, vol. 969, no. 1-3, pp. 1-12, 2011.

[7] K. Sadasivam and R. Kumaresan, "Theoretical investigation on the antioxidant behavior of chrysoeriol and hispidulin flavonoid compounds-a DFT study," Computational and Theoretical Chemistry, vol. 963, no. 1, pp. 227-235, 2011.

[8] D. Mikulski, M. Szelaogonekg, M. Molski, and R. Górniak, "Quantum-chemical study on the antioxidation mechanisms of trans-resveratrol reactions with free radicals in the gas phase, water and ethanol environment," Journal of Molecular Structure: THEOCHEM, vol. 951, no. 1-3, pp. 37-48, 2010.

[9] J. Rimarčík, V. Lukeš, E. Klein, and M. Ilčin, "Study of the solvent effect on the enthalpies of homolytic and heterolytic 
$\mathrm{N}-\mathrm{H}$ bond cleavage in $p$-phenylenediamine and tetracyano- $p$ phenylenediamine," Journal of Molecular Structure, vol. 952, no. $1-3$, pp. 25-30, 2010.

[10] J. H. Fellman, T. S. Fujita, and E. S. Roth, "Substrate specificity of p-hydroxyphenylpyruvate hydroxylase," Biochimica et Biophysica Acta, vol. 268, no. 2, pp. 601-604, 1972.

[11] S. Lindstedt and M. Rundgren, "Blue color, metal content, and substrate binding in 4-hydroxyphenylpyruvate dioxygenase from Pseudomonas sp. strain P.J. 874," The Journal of Biological Chemistry, vol. 257, no. 20, pp. 11922-11931, 1982.

[12] M. Nsangou, J. J. Fifen, Z. Dhaouadi, and S. Lahmar, "Hydrogen atom transfer in the reaction of hydroxycinnamic acids with radical $\cdot \mathrm{OH}$ and radical $\cdot \mathrm{HO}_{2}$ radicals: DFT study, Journal of Molecular Structure, vol. 862, no. 1-3, pp. 53-59, 2008.

[13] M. R. Alberto, M. E. Farías, and M. C. M. de Nadra, "Effect of gallic acid and catechin on Lactobacillus hilgardii $5 \mathrm{w}$ growth and metabolism of organic compounds," Journal of Agricultural and Food Chemistry, vol. 49, no. 9, pp. 4359-4363, 2001.

[14] M. R. Alberto, M. E. Farías, and M. C. Manca de Nadra, "Effect of wine phenolic compounds on Lactobacillus hilgardii 5w viability," Journal of Food Protection, vol. 65, no. 1, pp. 211213, 2002.

[15] M. Alberto, C. Gomez-Cordoves, and M. M. de Nadra, "Metabolism of gallic acid and catechin by Lactobacillus hilgardii from wine," Journal of Agricultural and Food Chemistry, vol. 52, no. 21, pp. 6465-6469, 2004.

[16] M. J. Rodríguez Vaquero, M. R. Alberto, and M. C. Manca de Nadra, "Influence of phenolic compounds from wines on the growth of Listeria monocytogenes," Food Control, vol. 18, no. 5, pp. 587-593, 2007.

[17] H. Milane, La quercétine et ses dérivés: molécules à caractère pro-oxydant ou capteurs de radicaux libres; études et applications thérapeutiques [Ph.D. thesis], Université Louis PasteurStrasbourg I, 2004.

[18] J. J. Fifen, M. Nsangou, Z. Dhaouadi, O. Motapon, and S. Lahmar, "Single or double hydrogen atom transfer in the reaction of metal—associated phenolic acids with ${ }^{\circ} \mathrm{OH}$ radical: DFT Study," Journal of Molecular Structure: THEOCHEM, vol. 901, no. 1-3, pp. 49-55, 2009.

[19] D. M. Miller, G. R. Buettner, and S. D. Aust, "Transition metals as catalysts of autoxidation reactions," Free Radical Biology and Medicine, vol. 8, no. 1, pp. 95-108, 1990.

[20] E. Cadenas and H. Sies, “The lag phase," Free Radical Research, vol. 28, no. 6, pp. 601-609, 1998.

[21] N. Pastor, H. Weinstein, E. Jamison, and M. Brenowitz, "A detailed interpretation of $\mathrm{OH}$ radical footprints in a TBPDNA complex reveals the role of dynamics in mechanism of sequence-specific binding," Journal of Molecular Biology, vol. 304, no. 1, pp. 55-68, 2000.

[22] T. N. Truong and D. G. Truhlar, "Ab initio transition state theory calculations of the reaction rate for $\mathrm{OH}+\mathrm{CH}_{4} \rightarrow \mathrm{H}_{2} \mathrm{O}+\mathrm{CH}_{3}$," The Journal of Chemical Physics, vol. 93, no. 3, pp. 1761-1769, 1990.

[23] R. T. Skodje and D. G. Truhlar, "Parabolic tunneling calculations," The Journal of Physical Chemistry, vol. 85, no. 6, pp. 624628, 1981.

[24] E. Wigner, "Crossing of potential thresholds in chemical reactions," Zeitschrift für Physikalische Chemie B, vol. 19, pp. 203216, 1932.

[25] D. G. Truhlar and A. Kuppermann, "Exact tunneling calculations," Journal of the American Chemical Society, vol. 93, no. 8, pp. 1840-1851, 1971.
[26] D. G. Truhlar and A. Kuppermann, "A test of transition state theory against exact quantum mechanical calculations," Chemical Physics Letters, vol. 9, no. 3, pp. 269-272, 1971.

[27] C. Eckart, "The penetration of a potential barrier by electrons," Physical Review, vol. 35, no. 11, pp. 1303-1309, 1930.

[28] J. Zheng, S. Zhang, B. J. Lynch et al., POLYRATE-version 2010, 2010.

[29] J. Tomasi, B. Mennucci, and R. Cammi, "Quantum mechanical continuum solvation models," Chemical Reviews, vol. 105, no. 8, pp. 2999-3093, 2005.

[30] M. J. Frisch, G. W. Trucks, H. B. Schlegel et al., Gaussian 03, Gaussian, Inc, Wallingford, Conn, USA, 2004.

[31] A. D. Becke, "Density-functional exchange-energy approximation with correct asymptotic behavior," Physical Review A, vol. 38, no. 6, pp. 3098-3100, 1988.

[32] C. Lee, W. Yang, and R. G. Parr, "Development of the ColleSalvetti correlation-energy formula into a functional of the electron density," Physical Review B, vol. 37, no. 2, pp. 785-789, 1988.

[33] V. A. Rassolov, M. A. Ratner, J. A. Pople, P. C. Redfern, and L. A. Curtiss, "6-31G* basis set for third-row atoms," Journal of Computational Chemistry, vol. 22, no. 9, pp. 976-984, 2001.

[34] M. J. Frisch, J. A. Pople, and J. S. Binkley, "Self-consistent molecular orbital methods 25. Supplementary functions for Gaussian basis sets," The Journal of Chemical Physics, vol. 80, no. 7, pp. 3265-3269, 1984.

[35] T. Clark, J. Chandrasekhar, G. W. Spitznagel, and P. V. R. Schleyer, "Efficient diffuse function-augmented basis sets for anion calculations. III. The $3-21+G$ basis set for first-row elements, Li-F," Journal of Computational Chemistry, vol. 4, no. 3, pp. 294-301, 1983.

[36] A. E. Reed, L. A. Curtiss, and F. Weinhold, "Intermolecular interactions from a natural bond orbital, donor-acceptor viewpoint," Chemical Reviews, vol. 88, no. 6, pp. 899-926, 1988.

[37] F. Weinhold and J. E. Carpenter, The Structure of Small Molecules and Ions, Plenum, 1988.

[38] L. A. Curtiss, K. Raghavachari, P. C. Redfern, V. Rassolov, and J. A. Pople, "Gaussian-3 (G3) theory for molecules containing first and second-row atoms," The Journal of Chemical Physics, vol. 109, no. 18, pp. 7764-7776, 1998.

[39] A. G. Baboul, L. A. Curtiss, P. C. Redfern, and K. Raghavachari, "Gaussian-3 theory using density functional geometries and zero-point energies," Journal of Chemical Physics, vol. 110, no. 16, pp. 7650-7657, 1999.

[40] A. Meffert and J. Grotemeyer, "Reactions in molecular clusters: proton transfer to small amino acids," European Journal of Mass Spectrometry, vol. 1, no. 1, pp. 594-598, 1995.

[41] A. Meffert and J. Grotemeyer, "Formation, stability and fragmentation of biomolecular clusters in a superasonic jet investigated with nano- and femtosecond laser pulses," Physical Chemistry Chemical Physics, vol. 102, no. 3, pp. 459-468, 1998.

[42] F. H. Yassin and D. S. Marynick, "Computational estimates of the gas-phase acidities of dihydroxybenzoic acid radical cations and their corresponding neutral species," Journal of Molecular Structure, vol. 629, pp. 223-235, 2003.

[43] M. Nsangou, Z. Dhaouadi, N. Jaidane, and Z. B. Lakhdar, "DFT study of the structure of hydroxybenzoic acids and their reactions with radical $\mathrm{OH}$ and ' $\mathrm{O}_{2}^{-}$radicals," Journal of Molecular Structure: THEOCHEM, vol. 850, no. 1-3, pp. 135$143,2008$. 
[44] Z. Dhaouadi, M. Nsangou, N. Garrab, E. H. Anouar, K. Marakchi, and S. Lahmar, "DFT study of the reaction of quercetin with $\mathrm{O}_{2}^{-}$and 'OH radicals," Journal of Molecular Structure, vol. 904, no. 1-3, pp. 35-42, 2009.

[45] J. E. Bartmess, "Thermodynamics of the electron and the proton," The Journal of Physical Chemistry, vol. 98, no. 25, pp. 6420-6424, 1994.

[46] J. J. Fifen, "Thermodynamics of the electron revisited and generalized," Journal of Chemical Theory and Computation, vol. 9, no. 7, pp. 3165-3169, 2013.

[47] J. J. Fifen, Z. Dhaouadi, and M. Nsangou, "Revision of the thermodynamics of the proton in gas phase," The Journal of Physical Chemistry A, vol. 118, no. 46, pp. 11090-11097, 2014.

[48] M. M. Bizarro, B. J. Costa Cabral, R. M. B. Borges dos Santos, and J. A. Martinho Simões, "Substituent effects on the $\mathrm{O}-\mathrm{H}$ bond dissociation enthalpies in phenolic compounds: agreements and controversies," Pure and Applied Chemistry, vol. 71, no. 7, pp. 1249-1256, 1999.

[49] V. D. Parker, "Homolytic bond (H-A) dissociation free energies in solution. Applications of the standard potential of the (H+/H.bul.) couple," Journal of the American Chemical Society, vol. 114, no. 19, pp. 7458-7462, 1992.

[50] E. Wilhelm and R. Battino, "Thermodynamic functions of the solubilities of gases in liquids at $25^{\circ} \mathrm{C}$," Chemical Reviews, vol. 73, no. 1, pp. 1-9, 1973.

[51] M. D. Tissandier, K. A. Cowen, W. Y. Feng et al., “The proton's absolute aqueous enthalpy and Gibbs free energy of solvation from cluster-ion solvation data," The Journal of Physical Chemistry A, vol. 102, no. 40, pp. 7787-7794, 1998.

[52] J. Jortner and R. M. Noyes, "Some thermodynamic properties of the hydrated electron," Journal of Physical Chemistry, vol. 70, no. 3, pp. 770-774, 1966.

[53] J. M. Mayer, D. A. Hrovat, J. L. Thomas, and W. T. Borden, "Proton-coupled electron transfer versus hydrogen atom transfer in benzyl/toluene, methoxyl/methanol, and phenoxyl/phenol self-exchange reactions," Journal of the American Chemical Society, vol. 124, no. 37, pp. 11142-11147, 2002.

[54] S. Hammes-Schiffer and N. Iordanova, "Theoretical studies of proton-coupled electron transfer reactions," Biochimica et Biophysica Acta, vol. 1655, no. 1-3, pp. 29-36, 2004. 

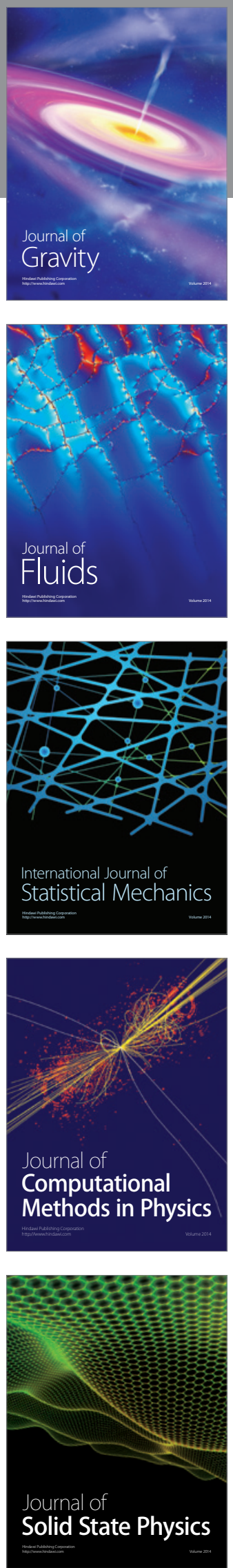

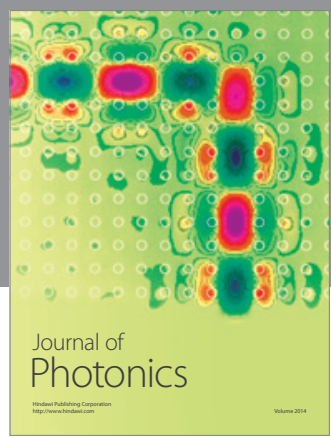

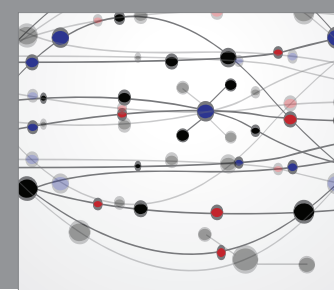

The Scientific World Journal

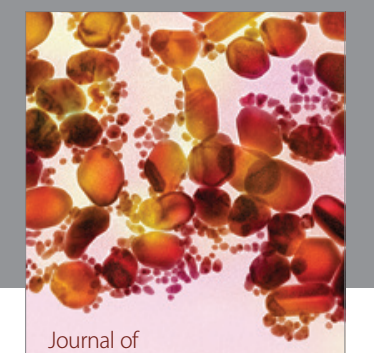

Soft Matter
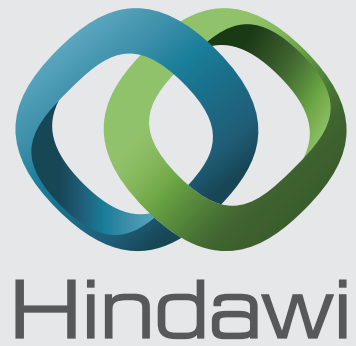

Submit your manuscripts at

http://www.hindawi.com
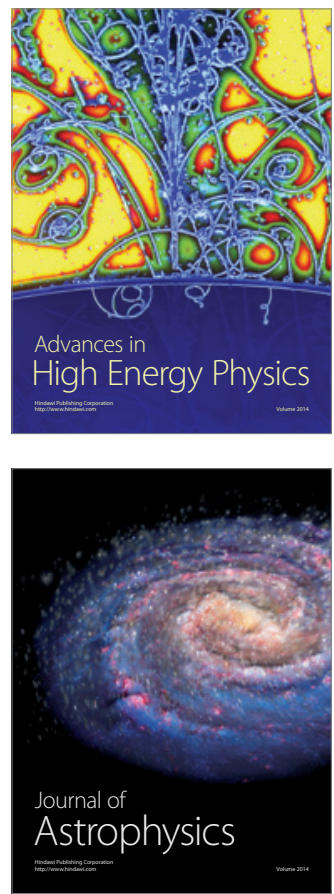
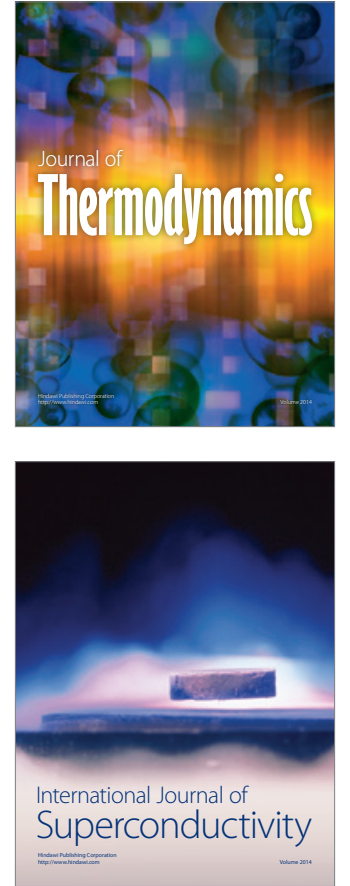
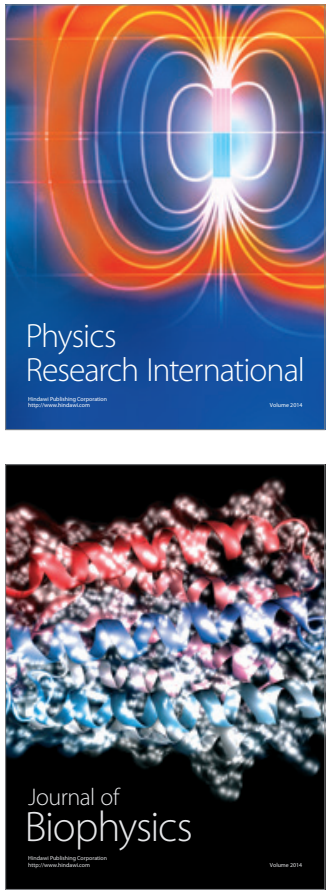
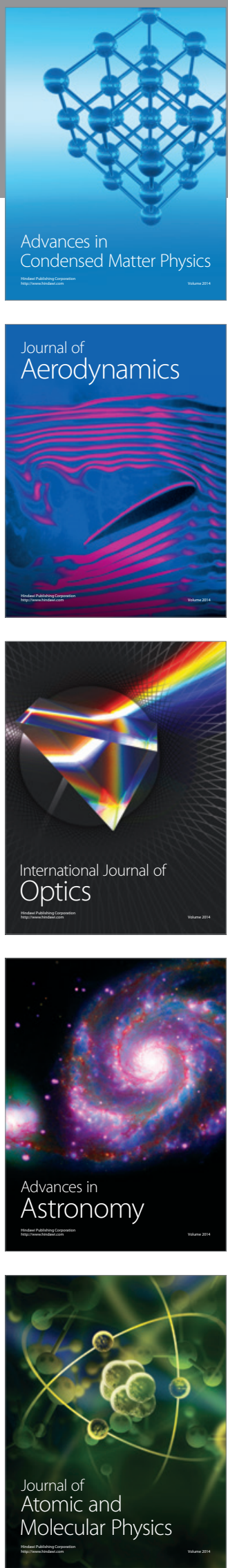